\title{
Decay properties for the solutions of a partial differential equation with memory
}

\author{
JAN PRÜSS
}

\begin{abstract}
Decay properties in energy norm for solutions of a class of partial differential equations with memory are studied by means of frequency domain methods. Our results are optimal for this class, as we are able to characterize polynomial as well as exponential decay rates. The results apply to models for viscoelastic materials. An extension to a semilinearly perturbed problem is also included.
\end{abstract}

Mathematics Subject Classification (2000). 34K20,45K05.

Keywords. Partial differential equations with memory, stability, decay properties, frequency domain methods.

1. Introduction and main results. Let $X$ be a Hilbert space, $A$ an unbounded, selfadjoint, positive definite operator in $X$ and let $k \in L_{1}\left(\mathbb{R}_{+}\right)$be a scalar memory kernel. In this paper we consider the problem

$$
\begin{aligned}
\ddot{u}(t)+A u(t)-(k * A u)(t) & =f(t), \quad t>0, \\
u(0)=u_{0}, \quad \dot{u}(0) & =u_{1} .
\end{aligned}
$$

Here $f: \mathbb{R}_{+} \rightarrow X$ as well as $u_{0}, u_{1} \in X$ are given data, a dot means time derivative, and the star indicates the convolution, i.e.

$$
(k * u)(t)=\int_{0}^{t} k(t-s) u(s) d s, \quad t \geq 0 .
$$

This problem arises in several applied fields, like viscoelasticity or heat conduction with memory, and in such applications the operator $A$ typically is the negative Laplacian in $X=L_{2}(\Omega)$, or the elasticity operator, the Stokes operator, or the biharmonic $\Delta^{2}$, etc. equipped with suitable boundary conditions. 
We consider in this paper stability properties of the solutions of (1.1), in particular decay of polynomial or exponential type. Typically, the kernel $k(t)$ is nonnegative nonincreasing, and satisfies

$$
\int_{0}^{\infty} k(t) d t<1 ;
$$

the latter is necessary for the well-posedness of the problem. To avoid degenerate cases, we also assume $k \in W_{1, l o c}^{1}(0, \infty)$, i.e. $k(t)$ is locally absolutely continuous on $(0, \infty)$. However, in contrast to other recent papers, we allow $k(t)$ to be singular at $t=0$; an example is the standard kernel

$$
k(t)=k_{0} \frac{t^{\beta-1}}{\Gamma(\beta)} e^{-\gamma t}, \quad t>0,
$$

where $\gamma>0, \beta \in(0,1)$ and $0<k_{0}<\gamma^{\beta}$. It is shown in Section 3 that under these assumptions, the solutions are asymptotically stable in energy norm, which means (in case $f \equiv 0$ ) that the solution $u$ satisfies

$$
\dot{u}, A^{1 / 2} u \in L_{1}\left(\mathbb{R}_{+} ; X\right) \cap C_{0}\left(\mathbb{R}_{+} ; X\right) .
$$

These assertions more or less already follow from results in my book [8], Section 10, but are definitely contained in the paper [4]. However, since [4] is not easily accessible, we give a direct and simple proof here.

On the other hand, neither in my book [8] nor in the papers [4], [5] stronger decay properties are considered. It is a common belief that to obtain exponential or polynomial decay one has to use the method of Ljapunov functionals. It is the main emphasis in this paper to show that such decay estimates can be obtained by means of frequency domain methods, with similar effort as for the basic asymptotic stability result.

Concerning polynomial decay, we prove in Section 4 decay rates like

$$
|\dot{u}(t)|+\left|A^{1 / 2} u(t)\right| \leq C t^{-m} \quad \text { as } t \rightarrow \infty,
$$

provided the moment condition $\int_{0}^{\infty} t^{m} k(t) d t<\infty$ is imposed on the kernel $k(t)$. This condition is shown to be optimal if one considers instead of (1.3) the property

$$
t^{m} \dot{u}, t^{m} A^{1 / 2} u \in L_{1}\left(\mathbb{R}_{+} ; X\right), \quad \text { for all } u_{0} \in D\left(A^{1 / 2}\right), u_{1} \in X .
$$

Section 5 is devoted to exponential decay in energy norm. Assuming that the kernel $k(t)$ has exponential decay, we show that the solution $u(t)$ has an exponential decay in energy norm as well, and we are able to obtain the optimal decay rate. On the other hand, we also show that exponential decay of the solutions implies exponential decay of the kernel.

Finally in Section 6 we apply our results to the semilinearly perturbed problem

$$
\begin{aligned}
\ddot{u}(t)+A u(t)-(k * A u)(t) & =f(u(t), \dot{u}(t)), \quad t>0, \\
u(0)=u_{0}, \quad \dot{u}(0) & =u_{1},
\end{aligned}
$$


where $f: D\left(A^{1 / 2}\right) \times X \rightarrow X$ is Lipschitz in a neighborhood of 0 , with $f(0)=0$ and a sufficiently small Lipschitz constant.

In this paper we advertise for frequency domain methods, which are appropriate due to the presence of a convolution memory term and the Hilbert space setting. Plancherel's theorem is available and gives optimal results for the problem in question. In particular, our results are natural and more precise than those available in the literature, cf. $[6,7,2,3,1]$, in fact, they are optimal.

2. Well-posedness and energy estimates. Integrating (1.1) twice we obtain the equivalent problem

$$
u(t)+(a * A u)(t)=u_{0}+t u_{1}+(t * f)(t), \quad t \geq 0,
$$

where

$$
a(t)=t-t * k(t)=\left(1-b_{0}\right) t+(1 * b)(t), \quad b(t)=\int_{t}^{\infty} k(s) d s, t \geq 0,
$$

and $b_{0}:=b(0)=\int_{0}^{\infty} k(s) d s<1$. Since $b(t)$ is nonnegative, nonincreasing and convex, we see that $b(t)$ as well as $d a(t)=\dot{a}(t) d t$ are of positive type, see [8, Proposition 3.3]. Therefore we may apply [8, Corollary 1.2], to see that problem (1.1) is well-posed in $X$. The solution is given by the variation of parameters formula

$$
u(t)=S(t) u_{0}+R(t) u_{1}+(R * f)(t), \quad t \geq 0,
$$

where $S(t)$ is the resolvent of (1.1) defined by the operator equation

$$
S(t)+A(a * S)(t)=I, \quad t \geq 0,
$$

and $R(t)=\int_{0}^{t} S(\tau) d \tau$ its integral. Thus we only need to study the properties of $S(t)$ and $R(t)$. From [8, Corollary 1.2], we have $|S(t)| \leq 1$, for all $t \geq 0$. Note that since $k(t)$ is real and $A$ is selfadjoint, $S(t)$ and $R(t)$ are selfadjoint as well.

To obtain the energy estimates, we let $f \equiv 0$, and note that $S(t)$ as well as $R(t)$ commute with $A$. Hence for $u_{0}, u_{1} \in D(A)$ we have $u \in C^{2}\left(\mathbb{R}_{+} ; X\right) \cap C\left(\mathbb{R}_{+} ; D(A)\right)$, and also $u \in C^{1}\left(\mathbb{R}_{+} ; D\left(A^{1 / 2}\right)\right)$. We first rewrite

$$
A u(t)-(k * A u)(t)=\left(1-b_{0}\right) A u(t)+A(b * \dot{u})(t)+b(t) A u_{0},
$$

then multiply (1.1) (with $f \equiv 0$ ) by $\dot{u}$. This implies

$$
\frac{1}{2} \frac{d}{d t}\left[|\dot{u}|^{2}+\left(1-b_{0}\right)\left|A^{1 / 2} u\right|^{2}\right]+\left(b * A^{1 / 2} \dot{u} \mid A^{1 / 2} \dot{u}\right)=-b(t)\left(A u_{0} \mid \dot{u}(t)\right) .
$$

Integration of this identity yields

$$
\begin{aligned}
& |\dot{u}(t)|^{2}+\left(1-b_{0}\right)\left|A^{1 / 2} u(t)\right|^{2}+2 \int_{0}^{t}\left(b * A^{1 / 2} \dot{u}(s) \mid A^{1 / 2} \dot{u}(s)\right) d s \\
& =\left|u_{1}\right|^{2}+\left(1-b_{0}\right)\left|A^{1 / 2} u_{0}\right|^{2}-2 \int_{0}^{t} b(s)\left(A^{1 / 2} u_{0} \mid A^{1 / 2} \dot{u}(s)\right) d s .
\end{aligned}
$$


If $u_{0}=0$ then $u(t)=R(t) u_{1}$, hence since $b(t)$ is of positive type we obtain

$$
\left|A^{1 / 2} R(t)\right| \leq 1 / \sqrt{1-b_{0}}, \quad t \geq 0 .
$$

On the other hand, if $u_{1}=0$ then $u(t)=S(t) u_{0}$, hence

$$
|\dot{u}(t)|^{2}+\left(1-b_{0}\right)\left|A^{1 / 2} u(t)\right|^{2} \leq\left(1-b_{0}\right)\left|A^{1 / 2} u_{0}\right|^{2}-2 \int_{0}^{t} b(s)\left(A^{1 / 2} \dot{u}(s) \mid A^{1 / 2} u_{0}\right) d s,
$$

which with an integration by parts yields

$$
\begin{aligned}
& |\dot{u}(t)|^{2}+\left(1-b_{0}\right)\left|A^{1 / 2} u(t)\right|^{2} \leq\left(1+b_{0}\right)\left|A^{1 / 2} u_{0}\right|^{2}+ \\
& +2 b(t)\left|A^{1 / 2} u(t)\right|\left|A^{1 / 2} u_{0}\right|+2 \int_{0}^{t} k(s)\left|A^{1 / 2} u(s)\right|\left|A^{1 / 2} u_{0}\right| d s \\
& \leq\left(1+b_{0}\right)\left|A^{1 / 2} u_{0}\right|^{2}+2 b_{0}\left|A^{1 / 2} u_{0}\right|^{2}+2 \int_{0}^{\infty} k(s) d s\left|A^{1 / 2} u_{0}\right|^{2} \\
& =\left(1+5 b_{0}\right)\left|A^{1 / 2} u_{0}\right|^{2},
\end{aligned}
$$

since $A^{1 / 2} u(t)=A^{1 / 2} S(t) u_{0}=S(t) A^{1 / 2} u_{0}$ which yields $\left|A^{1 / 2} u(t)\right| \leq\left|A^{1 / 2} u_{0}\right|$, for $|S(t)| \leq 1$. This implies

$$
\left|\dot{S}(t) A^{-1 / 2}\right| \leq \sqrt{1+5 b_{0}}, \quad t \geq 0,
$$

and then by $\ddot{S}(t) A^{-1}=-S(t)+(k * S)(t)$

$$
\left|\ddot{S}(t) A^{-1}\right| \leq 1+b_{0}, \quad t \geq 0 .
$$

Thus we have the following result.

Proposition 2.1. Let $A$ be selfadjoint positive definite in the Hilbert space $X$, and let $k \in L_{1}\left(\mathbb{R}_{+}\right)$be nonnegative nonincreasing and such that $\int_{0}^{\infty} k(s) d s<1$.

Then problem (1.1) is well-posed in $X$, and the solution is given by the variation of parameters formula (2.2). The resolvent $S(t)$ and its integral $R=1 * S$ satisfy

$$
\begin{aligned}
|S(t)| & \leq 1, \quad\left|A^{1 / 2} R(t)\right| \leq 1 / \sqrt{1-b_{0}}, & & t \geq 0, \\
\left|\dot{S}(t) A^{-1 / 2}\right| & \leq \sqrt{1+5 b_{0}}, \quad\left|\ddot{S}(t) A^{-1}\right| \leq 1+b_{0}, & & t \geq 0 .
\end{aligned}
$$

Each of these operator families is strongly continuous on $\mathbb{R}_{+}$. In particular, $u(t)$ is a strong solution of (1.1) if $u_{0} \in D(A), u_{1} \in D\left(A^{1 / 2}\right)$ and $f=f_{1}+f_{2}$, with $f_{1} \in W_{1, l o c}^{1}\left(\mathbb{R}_{+} ; X\right)$ and $f_{2} \in C\left(\mathbb{R}_{+} ; D\left(A^{1 / 2}\right)\right)$.

3. Asymptotic stability. As shown in [8, Section 10], the principal asymptotic stability property of evolutionary integral equations like (2.1) is the strong integrability of its resolvent, i.e.

$$
S(\cdot) x \in L_{1}\left(\mathbb{R}_{+} ; X\right), \quad \text { for all } x \in X .
$$


To derive this property we therefore may employ, [8, Corollary 10.5]. The properties of the kernels $k(t)$ under consideration here imply that the assumptions of this result are satisfied. Since $S(t)$ and $\dot{S}(t) A^{-1 / 2}$ are bounded, we also obtain $S(\cdot) x \in$ $C_{0}\left(\mathbb{R}_{+} ; X\right)$, by the Banach-Steinhaus theorem.

However, since in this paper we are concerned with the energy norm, we also need strong integrability of $A^{1 / 2} R(t)$ and $\dot{S}(t) A^{-1 / 2}$.

The main result of this section reads as follows.

Theorem 3.1. Let $A$ be a selfadjoint positive definite operator in the Hilbert space $X$, and let $k \in L_{1}\left(\mathbb{R}_{+}\right) \cap W_{1, \text { loc }}^{1}(0, \infty)$ be nonnegative, nonincreasing and not identically 0 ; assume the well-posedness condition $\int_{0}^{\infty} k(t) d t<1$.

Then the operator families $S(t), A^{1 / 2} R(t), \dot{S}(t) A^{-1 / 2}$ and $\ddot{S}(t) A^{-1}$ are strongly integrable, and converge strongly to 0 as $t \rightarrow \infty$.

Proof. (a) We define

$$
K(\lambda):=\widehat{R}(\lambda)=\left(\lambda^{2}+(1-\widehat{k}(\lambda)) A\right)^{-1}, \quad \operatorname{Re} \lambda>0,
$$

where the hat indicates the Laplace transform. Since $R(t)$ is uniformly bounded, $K(\lambda)$ is well-defined and holomorphic on $\mathbb{C}_{+}=\{\lambda \in \mathbb{C}: \operatorname{Re} \lambda>0\}$. In the first step we extend $K(\lambda)$ to the imaginary axis. For this purpose we consider the problem

$$
-\rho^{2} v+(1-\widehat{k}(i \rho)) A v=g,
$$

where $\rho \in \mathbb{R}$ and $g \in X$ are given. For $\rho=0$ this problem is uniquely solvable since $A$ is positive definite and $\widehat{k}(0)=b_{0}<1$, we have $K(0)=\left(1-b_{0}\right)^{-1} A^{-1}$. So suppose $\rho \neq 0$. If we have a solution $v$ of (3.1), taking the inner product of (3.1) with $v$ and decomposing into real and imaginary parts we obtain

$$
\begin{aligned}
-\rho^{2}|v|^{2}+(1-\operatorname{Re} \widehat{k}(i \rho))\left|A^{1 / 2} v\right|^{2} & =\operatorname{Re}(g \mid v) \\
-\operatorname{Im} \widehat{k}(i \rho)\left|A^{1 / 2} v\right|^{2} & =\operatorname{Im}(g \mid v) .
\end{aligned}
$$

Using $|\widehat{k}(i \rho)| \leq \widehat{k}(0)=b_{0}$, the first identity implies by Young's inequality

$$
\frac{1}{2} \rho^{2}|v|^{2} \leq \frac{1}{2 \rho^{2}}|g|^{2}+\left(1+b_{0}\right)\left|A^{1 / 2} v\right|^{2} .
$$

Multiplying the second identity in (3.2) by $\rho$ this implies again by Young's inequality

$$
-\rho \operatorname{Im} \widehat{k}(i \rho)\left|A^{1 / 2} v\right|^{2} \leq\left(\frac{1}{2 \varepsilon}+\frac{\varepsilon}{2 \rho^{2}}\right)|g|^{2}+\frac{\varepsilon\left(1+b_{0}\right)}{2}\left|A^{1 / 2} v\right|^{2} .
$$

Next we have

$$
-\rho \operatorname{Im} \widehat{k}(i \rho)=\int_{0}^{\infty} k(t) \rho \sin (\rho t) d t=\int_{0}^{\infty}[-\dot{k}(t)][1-\cos (\rho t)] d t>0,
$$

unless $\dot{k} \equiv 0$, which means $k \equiv 0$. This shows that $-\rho^{2} /(1-\widehat{k}(i \rho)) \notin(0, \infty) \supset \sigma(A)$. Thus by positive definiteness of $A$ we see that $K(i \rho)=L(i \rho)^{-1}$ exists, and since 
$\widehat{k}(\lambda)$ is continuous on $\overline{\mathbb{C}}_{+}$the family $K(\lambda)$ is also continuous, even in operator norm on $\overline{\mathbb{C}}_{+}$.

Since $\dot{k}(t)$ belongs to $L_{1}(\eta, \infty)$, for each $\eta>0$, the Riemann-Lebesgue Lemma implies

$$
\liminf _{|\rho| \rightarrow \infty}[-\rho \operatorname{Im} \widehat{k}(i \rho)] \geq \liminf _{|\rho| \rightarrow \infty} \int_{\eta}^{\infty}[-\dot{k}(t)][1-\cos (\rho t)] d t=k(\eta),
$$

hence we obtain the lower bound

$$
\liminf _{|\rho| \rightarrow \infty}[-\rho \operatorname{Im} \widehat{k}(i \rho)] \geq k(0+)>0 ;
$$

note that $k(0+)=\infty$ if the kernel $k(t)$ is singular at $t=0$. Choosing $\varepsilon>0$ small enough, (3.4) implies that $\left|A^{1 / 2} v\right|$ is bounded by $|g|$, uniformly for $|\rho| \rightarrow \infty$. Continuity of $A^{1 / 2} K(i \rho)$ then shows that $\left|A^{1 / 2} K(i \rho)\right|$ is uniformly bounded on $\mathbb{R}$, and by (3.3) we see that $|\rho K(i \rho)|$ also has this property.

(b) In the next step we show that $A^{1 / 2} K(i \cdot) x$ and $i \rho K(i \cdot) x$ are in $L_{2}(\mathbb{R} ; X)$, for each $x \in X$. Plancherel's theorem then implies $A^{1 / 2} R(\cdot) x$ and $S(\cdot) x$ in $L_{2}\left(\mathbb{R}_{+} ; X\right)$, since $\widehat{S}(\lambda)=\lambda K(\lambda)$. For this purpose let $\omega>0$ be fixed. Since $A^{1 / 2} R(t)$ and $S(t)$ are uniformly bounded, we have $e^{-\omega t} A^{1 / 2} R(t) x$ and $e^{-\omega t} S(t) x$ in $L_{2}\left(\mathbb{R}_{+} ; X\right)$, for each $x \in X$, hence Plancherel's theorem yields $A^{1 / 2} K(\omega+i \cdot) x$ and $i \rho K(\omega+i \cdot) x$ in $L_{2}(\mathbb{R} ; X)$. By means of a resolvent formula we may write

$$
\begin{aligned}
K(i \rho) & =\frac{1-\widehat{k}(\omega+i \rho)}{1-\widehat{k}(i \rho)} K(\omega+i \rho)+K(i \rho)\left[\omega^{2}+2 i \omega \rho\right] K(\omega+i \rho) \\
& +K(i \rho) \frac{\rho^{2}(\widehat{k}(i \rho)-\widehat{k}(\omega+i \rho))}{1-\widehat{k}(i \rho)} K(\omega+i \rho) .
\end{aligned}
$$

Since $\int_{0}^{\infty} k(t) d t<1$, by the Paley-Wiener lemma (see, e.g., [8, Theorem 0.7]) there is a kernel $r \in L_{1}\left(\mathbb{R}_{+}\right)$such that $1 /(1-\widehat{k}(i \rho))=1+\widehat{r}(i \rho)$. This and the bounds on $K$ from (a) show that the first two terms in (3.5) multiplied by $A^{1 / 2}$ or by $\rho$ yield contributions in $L_{2}\left(\mathbb{R}_{;} X\right)$ when applied to an element $x \in X$. Concerning the last term observe that since $k \in L_{1}$ is nonnegative nonincreasing, we have $t \dot{k}(t)$ in $L_{1}\left(\mathbb{R}_{+}\right)$as well. Therefore, we obtain with an integration by parts

$$
i \rho(\widehat{k}(i \rho)-\widehat{k}(\omega+i \rho))=\int_{0}^{\infty} e^{-i \rho t}\left[\dot{k}(t)\left(1-e^{-\omega t}\right)+\omega k(t) e^{-\omega t}\right] d t,
$$

since $-t \dot{k}(t)$ is integrable, hence estimating we obtain

$$
|\rho(\widehat{k}(i \rho)-\widehat{k}(\omega+i \rho))| \leq 2 \omega b_{0} .
$$

Since bounded measurable functions are pointwise multipliers for $L_{2}$, this estimate shows that also the last term in (3.5) gives rise to a contribution in $L_{2}(\mathbb{R} ; X)$. 
(c) We finally show that $A^{1 / 2} R(t) x$ and $S(t) x$ are in $L_{1}\left(\mathbb{R}_{+} ; X\right)$; since

$$
\dot{S}(t) A^{-1 / 2}=-A^{1 / 2} R(t)+k * A^{1 / 2} R(t), \quad \ddot{S}(t) A^{-1}=-S(t)+k * S(t),
$$

the theorem is then proved. For this purpose we set $T(t):=R(t)-(k * R)(t)=$ $-\dot{S}(t) A^{-1}$; since $R(t)=T(t)+(r * T)(t)$ with $r \in L_{1}\left(\mathbb{R}_{+}\right)$as in (b), we see that it is sufficient to show $A^{1 / 2} T(t) x$ and $\dot{T}(t) x$ in $L_{1}\left(\mathbb{R}_{+} ; X\right)$ for each $x \in X$. Note that by (b) we already know these functions to belong to $L_{2}\left(\mathbb{R}_{+} ; X\right)$. Since $-t \dot{k}(t)$ belongs to $L_{1}\left(\mathbb{R}_{+}\right)$, by the identity

$$
\lambda \widehat{k}^{\prime}(\lambda)=-\widehat{\dot{k}}(\lambda)-\widehat{k}(\lambda),
$$

we see that $\lambda \widehat{k}^{\prime}(\lambda)$ is bounded on $\overline{\mathbb{C}}_{+}$, hence $\widehat{T}(i \rho)$ is differentiable and

$$
\widehat{T}^{\prime}(i \rho)=-K(i \rho)\left[2(1-\widehat{k}(i \rho))+i \rho \widehat{k}^{\prime}(i \rho)\right] i \rho K(i \rho) .
$$

From this we obtain $A^{1 / 2} \widehat{T}^{\prime}(i \rho) x$ and $i \rho \widehat{T}^{\prime}(i \rho) x$ in $L_{2}(\mathbb{R} ; X)$ for each $x \in X$, hence Plancherel's theorem implies $t A^{1 / 2} T(t) x$ and $t \dot{T}(t) x$ in $L_{2}\left(\mathbb{R}_{+} ; X\right)$. But then the estimate

$$
\begin{aligned}
\int_{0}^{\infty}\left|A^{1 / 2} T(t) x\right| d t & =\int_{0}^{1}\left|A^{1 / 2} T(t) x\right| d t+\int_{1}^{\infty}\left|t A^{1 / 2} T(t) x\right| d t / t \\
& \leq\left(\int_{0}^{\infty}\left|A^{1 / 2} T(t) x\right|^{2} d t\right)^{1 / 2}+\left(\int_{0}^{\infty}\left|t A^{1 / 2} T(t) x\right|^{2} d t\right)^{1 / 2}
\end{aligned}
$$

shows $A^{1 / 2} T(\cdot) x \in L_{1}\left(\mathbb{R}_{+} ; X\right)$, for each $x \in X$. In the same way $\dot{T}(\cdot) x \in L_{1}\left(\mathbb{R}_{+} ; X\right)$ is shown. The proof is now complete.

4. Polynomial decay. In recent years there has been considerable interest in deacy estimates for solutions of problems like (1.1). We show in this section how polynomial decay estimates can be derived by frequency domain methods, assuming just some moment conditions on the kernel $k(t)$.

For this purpose we first consider $T(t)$ defined as above by means of $T=$ $R-k * R$. Note that $T(0)=0$, hence $\lambda \widehat{T}(\lambda)=\widehat{\dot{T}}(\lambda)$. The Laplace transform of $T(t)$ is given by

$$
\widehat{T}(\lambda)=\left(\lambda^{2} /(1-\widehat{k}(\lambda))+A\right)^{-1}, \quad \operatorname{Re} \lambda \geq 0 .
$$

Differentiating we obtain with $\phi(\lambda)=\lambda^{2} /(1-\widehat{k}(\lambda))$

$$
\widehat{t T}(\lambda)=-\widehat{T}^{\prime}(\lambda)=\widehat{T}(\lambda)\left[\phi^{\prime}(\lambda)\right] \widehat{T}(\lambda) .
$$

Since $T(t)$ is selfadjoint this implies

$$
\left(t A^{1 / 2} T(t) x \mid x^{*}\right)=\int_{0}^{t}\left(A^{1 / 2} T(t-s) x^{*} \mid(d l * \dot{T} x)(s)\right) d s,
$$


where

$$
\widehat{d l}(\lambda)=\frac{2-3 \widehat{k}(\lambda)-\widehat{t \dot{k}}(\lambda)}{(1-\widehat{k}(\lambda))^{2}}=(1+\widehat{r}(\lambda))^{2}(2-3 \widehat{k}(\lambda)-\widehat{t \dot{k}}(\lambda))
$$

is the Laplace transform of a finite measure $d l$ supported on $\mathbb{R}_{+}$. Therefore

$$
\left|\left(t A^{1 / 2} T(t) x \mid x^{*}\right)\right| \leq\left|A^{1 / 2} T(\cdot) x^{*}\right|_{L_{2}}|d l|_{1}|\dot{T}(\cdot) x|_{L_{2}} \leq M_{1}|x|\left|x^{*}\right|,
$$

which shows $\left|t A^{1 / 2} T(t)\right| \leq M_{1}$ on $\mathbb{R}_{+}$. Similarly, we also see $|t \dot{T}(t)| \leq M_{2}$ on $\mathbb{R}_{+}$, with some constant $M_{2}>0$.

If in addition we have $\int_{0}^{\infty} t k(t) d t<\infty$ another differentiation yields

$$
\widehat{T}^{\prime \prime}(\lambda)=2 \widehat{T}(\lambda) \phi^{\prime}(\lambda) \widehat{T}(\lambda) \phi^{\prime}(\lambda) \widehat{T}(\lambda)-\widehat{T}(\lambda) \phi^{\prime \prime}(\lambda) \widehat{T}(\lambda) .
$$

Note that $t^{m} k \in L_{1}\left(\mathbb{R}_{+}\right)$implies $t^{m+1} \dot{k} \in L_{1}\left(\mathbb{R}_{+}\right)$as well as $t^{m+1} k(t) \rightarrow 0$ as $t \rightarrow \infty$. In fact, an integration by parts shows

$$
N^{m+1} k(N)-\int_{0}^{N} t^{m+1} \dot{k}(t) d t=(m+1) \int_{0}^{N} t^{m} k(t) d t \leq(m+1)\left|t^{m} k\right|_{L_{1}}<\infty,
$$

hence $t^{m+1} \dot{k} \in L_{1}\left(\mathbb{R}_{+}\right)$, and

$$
N^{m+1} k(N)=-N^{m+1} \int_{N}^{\infty} \dot{k}(t) d t \leq-\int_{N}^{\infty} t^{m+1} \dot{k}(t) d t \rightarrow 0 \text { as } N \rightarrow \infty .
$$

Since $\lambda \widehat{k}^{\prime \prime}(\lambda)=2 \widehat{t k}(\lambda)+\widehat{t^{2} \dot{k}}(\lambda)$ and $t k \in L_{1}\left(\mathbb{R}_{+}\right)$yields $t^{2} \dot{k} \in L_{1}\left(\mathbb{R}_{+}\right)$, we obtain in the same way as above boundedness of $t^{2} A^{1 / 2} T(t)$ and $t^{2} \dot{T}(t)$ on $\mathbb{R}_{+}$. Repeating these arguments, by induction we see that $t^{m-1} k \in L_{1}\left(\mathbb{R}_{+}\right)$implies that $t^{m} A^{1 / 2} T(t)$ and $t^{m} \dot{T}(t)$ are uniformly bounded on $\mathbb{R}_{+}$.

Looking now at $S(t)$, we have

$$
\widehat{S}(\lambda)=\lambda \widehat{T}(\lambda) /(1-\widehat{k}(\lambda)) .
$$

Differentiating this identity we get

$$
\widehat{S}^{\prime}(\lambda)=\left[(1-\widehat{k}(\lambda)) \widehat{\dot{T}}^{\prime}(\lambda)+\lambda \widehat{k}^{\prime}(\lambda) \widehat{T}(\lambda)\right] /(1-\widehat{k}(\lambda))^{2} .
$$

This yields $t S(t)$ bounded on $\mathbb{R}_{+}$, without further assumptions. By induction we then see that $t^{m-1} k \in L_{1}\left(\mathbb{R}_{+}\right)$implies $t^{m} S(t)$ uniformly bounded on $\mathbb{R}_{+}$.

On the other hand, looking at $A^{1 / 2} R(t)$ we get

$$
\widehat{R}^{\prime}(\lambda)=\frac{1}{1-\widehat{k}(\lambda)} \widehat{T}^{\prime}(\lambda)+\frac{\widehat{k}^{\prime}(\lambda)}{(1-\widehat{k}(\lambda))^{2}} \widehat{T}(\lambda) .
$$

Here we need $t k \in L_{1}\left(\mathbb{R}_{+}\right)$to conclude that $t A^{1 / 2} R(t)$ is uniformly bounded on $\mathbb{R}_{+}$. In this case induction yields $t^{m} A^{1 / 2} R(t)$ provided $t^{m} k \in L_{1}\left(\mathbb{R}_{+}\right)$. 
Finally, recall the relations

$$
\widehat{\dot{S}}(\lambda) A^{-1 / 2}=-(1-\widehat{k}(\lambda)) A^{1 / 2} \widehat{R}(\lambda)=-A^{1 / 2} \widehat{T}(\lambda),
$$

and

$$
\widehat{\ddot{S}}(\lambda) A^{-1}=-(1-\widehat{k}(\lambda)) \widehat{S}(\lambda)=-\widehat{\dot{T}}(\lambda) .
$$

Summarizing, we have shown the following result.

Theorem 4.1. Let the assumptions of Theorem 3.1 be satisfied. Assume in addition $\int_{0}^{\infty} t^{m} k(t) d t<\infty$ for some $m \geq 0$

Then there is a constant $M_{m}>0$ such that

$$
\left|t^{m+1} S(t)\right|,\left|t^{m+1} \dot{S}(t) A^{-1 / 2}\right|,\left|t^{m+1} \ddot{S}(t) A^{-1}\right|,\left|t^{m} A^{1 / 2} R(t)\right| \leq M_{m}, \quad t \geq 0 .
$$

The arguments in this section show in addition that e.g. $t^{m} A^{1 / 2} T(\cdot) x$ is in $L_{2}\left(\mathbb{R}_{+} ; X\right)$ if $t^{m-1} k \in L_{1}\left(\mathbb{R}_{+}\right)$. Therefore as in step (c) of Section 3, we get also $t^{m} A^{1 / 2} T(\cdot) x \in L_{1}\left(\mathbb{R}_{+} ; X\right)$ provided $t^{m} k \in L_{1}\left(\mathbb{R}_{+}\right)$. Analogous results hold for the other quantities in question, even for $t^{m} A^{1 / 2} R(t)$. These conditions are optimal. In fact, if $t R(\cdot) x$ is in $L_{1}\left(\mathbb{R}_{+} ; D\left(A^{1 / 2}\right)\right)$ for all $x \in X$ then $\widehat{R}^{\prime}(\lambda)$ is strongly continuous in $\mathcal{B}\left(X, D\left(A^{1 / 2}\right)\right)$ on $\overline{\mathbb{C}}_{+}$. In particular,

$$
\widehat{R}^{\prime}(0)=\widehat{k}^{\prime}(0) A K(0)^{2}
$$

exists. But since $\widehat{k}$ is differentiable at 0 if and only if $t k \in L_{1}\left(\mathbb{R}_{+}\right)$, this implies $t k \in L_{1}\left(\mathbb{R}_{+}\right)$.

We state the result following from these observation in the next corollary.

Corollary 4.2. Let the assumptions of Theorem 3.1 be satisfied. Assume in addition $\int_{0}^{\infty} t^{m} k(t) d t<\infty$ for some $m \geq 1$.

Then the operator families $t^{m} S(\cdot), t^{m} A^{1 / 2} R(\cdot), t^{m} \dot{S}(\cdot) A^{-1 / 2}, t^{m} \ddot{S}(\cdot) A^{-1}$ are strongly integrable and converge strongly to 0 as $t \rightarrow \infty$. The moment condition on $k$ is also necessary for these assertions.

5. Exponential decay. Assume now that there is $\alpha_{0}>0$ such that $\int_{0}^{\infty} e^{\alpha_{0} s} k(s) d s<$ $\infty$, which means that the kernel $k$ has exponential decay. In fact, since $k$ is nonnegative and nonincreasing we have

$$
\begin{aligned}
k(t) e^{\alpha_{0} t} & =k(t) e^{\alpha_{0} N}+k(t) \int_{N}^{t} \alpha_{0} e^{\alpha_{0} s} d s \\
& \leq k(t) e^{\alpha_{0} N}+\int_{N}^{\infty} \alpha e^{\alpha_{0} s} k(s) d s .
\end{aligned}
$$


This yields $k(t) e^{\alpha_{0} t} \rightarrow 0$ as $t \rightarrow \infty$. Similarly, an integration by parts yields

$$
\int_{\varepsilon}^{N}-\dot{k}(t) e^{\alpha_{0} t} d t=-\left.k(t) e^{\alpha_{0} t}\right|_{\varepsilon} ^{N}+\alpha_{0} \int_{\varepsilon}^{N} k(t) e^{\alpha_{0} t} d t,
$$

which shows that $\dot{k}(t) e^{\alpha_{0} t}$ is integrable over each interval $[\varepsilon, \infty)$. Thus we also obtain $t \dot{k} e^{\alpha t} \in L_{1}\left(\mathbb{R}_{+}\right)$, for each $\alpha<\alpha_{0}$.

Since $\widehat{k}(\lambda)$ is holomorphic in $\mathbb{C}_{-\alpha_{0}}:=\left\{\lambda \in \mathbb{C}: \operatorname{Re} \lambda>-\alpha_{0}\right\}$, we see that the operator-valued function $K(\lambda)$ extends holomorphically to a neighborhood of $i \mathbb{R}$. In the first step, we want to show that this neighborhood contains a strip $-\alpha<\operatorname{Re} \lambda<\alpha$, for some $\alpha>0$. For this we proceed as in (a) of the proof of Theorem 3.1.

(a) We choose $\alpha>0$ and let $\lambda=-\sigma+i \rho, 0<\sigma<\alpha$. Then $v=K(-\sigma+i \rho) g$ satisfies

$$
(-\sigma+i \rho)^{2} v+(1-\widehat{k}(-\sigma+i \rho)) A v=g .
$$

Multiplying this equation with $v$ and taking real and imaginary parts we obtain

$$
\begin{aligned}
\left(\sigma^{2}-\rho^{2}\right)|v|^{2}+(1-\operatorname{Re} \widehat{k}(-\sigma+i \rho))\left|A^{1 / 2} v\right|^{2} & =\operatorname{Re}(g \mid v), \\
\left.-2 \sigma \rho|v|^{2}-\operatorname{Im} \widehat{k}(-\sigma+i \rho)\right)\left|A^{1 / 2} v\right|^{2} & =\operatorname{Im}(g \mid v) .
\end{aligned}
$$

From these identities we obtain as in (a) of the proof of Theorem 3.1 the following estimate.

$$
\left(\rho^{2}-\sigma^{2}-\varepsilon\right)|v|^{2} \leq \frac{|g|^{2}}{4 \varepsilon}+(1-\operatorname{Re} \widehat{k}(-\sigma+i \rho))\left|A^{1 / 2} v\right|^{2} .
$$

Multiplying (5.1) with $\rho$ we get

$$
\begin{gathered}
-\rho \operatorname{Im} \widehat{k}(-\sigma+i \rho)\left|A^{1 / 2} v\right|^{2} \leq|g||\rho v|+2 \sigma \rho^{2}|v|^{2} \leq \frac{|g|^{2}}{4 \varepsilon}+(2 \sigma+\varepsilon)|\rho v|^{2} \\
\leq \frac{|g|^{2}}{4 \varepsilon}\left[1+\frac{\rho^{2}(2 \sigma+\varepsilon)}{\rho^{2}-\sigma^{2}-\varepsilon}\right]+\frac{\rho^{2}(2 \sigma+\varepsilon)}{\rho^{2}-\sigma^{2}-\varepsilon}[1-\operatorname{Re} \widehat{k}(-\sigma+i \rho)]\left|A^{1 / 2} v\right|^{2} .
\end{gathered}
$$

To obtain a lower bound for $-\rho \operatorname{Im} \widehat{k}(-\sigma+i \rho)$, we calculate

$$
\begin{aligned}
& -\rho \operatorname{Im} \widehat{k}(-\sigma+i \rho)=\int_{0}^{\infty}-\left[k(t) e^{\sigma t}\right](1-\cos (\rho t)) d t \\
& \geq \int_{\eta}^{\infty}[-\dot{k}(t)] e^{\sigma t}(1-\cos (\rho t)) d t-\sigma \int_{0}^{\infty} e^{\sigma t} k(t)(1-\cos (\rho t)) d t \\
& \rightarrow \int_{\eta}^{\infty}[-\dot{k}(t)] e^{\sigma t}-\sigma \int_{0}^{\infty} e^{\sigma t} k(t) d t= \\
& =k(\eta) e^{\sigma \eta}-\sigma \int_{0}^{\eta} k(t) e^{\sigma t} d t \geq k(\eta)-\alpha \int_{0}^{\eta} k(t) e^{\alpha t} d t,
\end{aligned}
$$


as $|\rho| \rightarrow \infty$, again employing the Riemann-Lebesgue lemma. This implies

$$
\liminf _{|\rho| \rightarrow \infty}-\rho \operatorname{Im} \widehat{k}(-\sigma+i \rho) \geq k(0+),
$$

uniformly for $0 \leq \sigma \leq \alpha_{0}$. Since $\widehat{k}(-\sigma+i \rho) \rightarrow 0$ uniformly w.r.t. $\sigma \in\left[0, \alpha_{0}\right]$ as well, as in (a) of the proof of Theorem 3.1 we may now conclude uniform bounds for $A^{1 / 2} K(\lambda)$ and $\lambda K(\lambda)$ in a strip of the form $-\alpha \leq \operatorname{Re} \lambda \leq 0$, provided $\alpha<\min \left\{\alpha_{0}, k(0+) / 2\right\}$.

(b) We look first again at $\widehat{T}(\lambda)=(\phi(\lambda)+A)^{-1}$, where $\phi(\lambda)=\lambda^{2} /(1-\widehat{k}(\lambda))$. We have

$$
\widehat{T}(-\sigma+i \rho)-\widehat{T}(\sigma+i \rho)=\widehat{T}(-\sigma+i \rho)[\phi(-\sigma+i \rho)-\phi(\sigma+i \rho)] \widehat{T}(\sigma+i \rho) .
$$

Since $\dot{T}(\cdot) e^{-\sigma t} x$ belongs to $L_{2}\left(\mathbb{R}_{+} ; X\right)$, as before Plancherel's theorem yields $i \rho \widehat{T}(\sigma+$ $i \cdot) x \in L_{2}(\mathbb{R} ; X)$, and then by boundedness of $A^{1 / 2} \widehat{T}(-\sigma+i \rho)$ and of $i \rho \widehat{T}(-\sigma+i \rho)$ proved in step (a) we obtain $A^{1 / 2} \widehat{T}(-\sigma+i \cdot) x$ and $i \rho \widehat{T}(-\sigma+i \cdot) x$ in $L_{2}(\mathbb{R} ; X)$ for each $x \in X$, provided $\phi(-\sigma+i \rho)-\phi(\sigma+i \rho)$ is bounded by $C(1+|\rho|)$. From this Plancherel's theorem implies $\dot{T}(\cdot) e^{\sigma t} x \in L_{2}\left(\mathbb{R}_{+} ; X\right)$ as well as $A^{1 / 2} T(\cdot) e^{\sigma t} x \in$ $L_{2}\left(\mathbb{R}_{+} ; X\right)$, for all $x \in X$, and then even

$$
|\dot{T}(t)|,\left|A^{1 / 2} T(t)\right| \leq M e^{-\sigma t}, \quad t \geq 0,
$$

as in the proof of Theorem 4.1. This yields uniform exponential decay of the relevant operator families $\dot{S}(t) A^{-1 / 2}=-A^{1 / 2} T(t)$, and $\ddot{S}(t) A^{-1}=-\dot{T}(t)$, but also of $A^{1 / 2} R(t)=A^{1 / 2} T(t)+\left(r * A^{1 / 2} T\right)(t), S(t)=\dot{T}(t)+(r * \dot{T})(t)$, as long as

$$
\widehat{k}(-\sigma)=\int_{0}^{\infty} k(t) e^{\sigma t}<1 .
$$

Thus it remains to estimate $\phi(-\sigma+i \rho)-\phi(\sigma+i \rho)$. We have

$$
\begin{aligned}
\phi(-\sigma+i \rho)-\phi(\sigma+i \rho) & =-\frac{4 i \sigma \rho}{1-\widehat{k}(\sigma+i \rho)} \\
& +(-\sigma+i \rho)^{2} \frac{\widehat{k}(-\sigma+i \rho)-\widehat{k}(\sigma+i \rho)}{(1-\widehat{k}(-\sigma+i \rho))(1-\widehat{k}(\sigma+i \rho))}
\end{aligned}
$$

and

$$
\begin{aligned}
& i \rho(\widehat{k}(-\sigma+i \rho)-\widehat{k}(\sigma+i \rho))=\int_{0}^{\infty} k(t) i \rho e^{-i \rho t}\left(e^{\sigma t}-e^{-\sigma t}\right) d t \\
& =\int_{0}^{\infty} \dot{k}(t) e^{-i \rho t}\left(e^{\sigma t}-e^{-\sigma t}\right) d t+\sigma \int_{0}^{\infty} k(t) e^{-i \rho t}\left(e^{\sigma t}+e^{-\sigma t}\right) d t .
\end{aligned}
$$


The latter implies

$$
|\rho(\widehat{k}(-\sigma+i \rho)-\widehat{k}(\sigma+i \rho))| \leq 4 \sigma \widehat{k}(-\sigma) \leq 4 \alpha_{0} \widehat{k}\left(-\alpha_{0}\right),
$$

and so we obtain

$$
|\phi(-\sigma+i \rho)-\phi(\sigma+i \rho)| \leq C(1+|\rho|), \quad \rho \in \mathbb{R},
$$

provided $\sigma \leq \alpha_{0}$.

Summarizing, we have proved the following result.

Theorem 5.1. Let the assumptions of Theorem 3.1 be satisfied. Assume in addition $\int_{0}^{\infty} e^{\alpha_{0} t} k(t) d t<\infty$ for some $\alpha_{0}>0$.

Then there are constants $\alpha>0$ and $M>0$ such that

$$
|S(t)|,\left|\dot{S}(t) A^{-1 / 2}\right|,\left|\ddot{S}(t) A^{-1}\right|,\left|A^{1 / 2} R(t)\right| \leq M e^{-\alpha t}, \quad t \geq 0 .
$$

The optimal decay rate $\gamma_{*}$ follows also from the proof of Theorem 5.1. To see this, suppose e.g. that for each $0<\gamma<\gamma_{*}$ there is a constant $M=M_{\gamma}$ such that $|R(t)| \leq M e^{-\gamma t}$, for $t>0$. Then the Laplace transform $\widehat{R}(\lambda)=K(\lambda)$ is holomorphic and bounded on each halfplane $\operatorname{Re} \lambda \geq-\gamma$. This implies as in $[8$, p. 50-51], that $\widehat{k}(\lambda)$ admits meromorphic extension to the halfplane $\operatorname{Re} \lambda>-\gamma_{*}$ and

$$
\phi(\lambda)=\lambda^{2} /(1-\widehat{k}(\lambda)) \notin \sigma(-A) \cup\{\infty\}, \quad \operatorname{Re} \lambda>-\gamma_{*} .
$$

In particular, we have $\widehat{k}(-\gamma)<1$, for all $\gamma<\gamma_{*}$. By positivity of $k(t)$, this implies $k(t) e^{\gamma t} \in L_{1}\left(\mathbb{R}_{+}\right)$, for each $\gamma<\gamma_{*}$. This yields the optimal decay rate $\gamma_{*}$ as

$$
\gamma_{*}=\min \left\{\alpha_{1}, \alpha_{2}\right\}
$$

where

$$
\alpha_{1}:=\sup \left\{\alpha>0: \int_{0}^{\infty} e^{\alpha t} k(t) d t<1\right\}
$$

and

$$
\alpha_{2}:=\sup \left\{\alpha>0: \phi\left(\mathbb{C}_{-\alpha}\right) \cap \sigma(-A)=\emptyset\right\} .
$$

What is the role of the condition $\alpha<k(0+) / 2$ in the proof of Theorem 5.1? Obviously, this is no restriction in the case of a singular kernel. However, if $k(t)$ is regular, then $\widehat{k}(\lambda) \sim k(0+) / \lambda$ for large $\lambda$, hence the solutions $\lambda(\mu)$ of the equation

$$
\lambda^{2}+\mu(1-\widehat{k}(\lambda))=0
$$

for large $\mu>0$ to first order asymptotically behave like $\lambda(\mu) \sim \pm i \sqrt{\mu}$, and to second order like

$$
\lambda(\mu) \sim \pm i \sqrt{\mu}(1-k(0+) / \lambda)^{1} / 2 \sim \pm i \sqrt{\mu}-k(0+) / 2 .
$$

Thus $\alpha_{2} \leq k(0+) / 2$ in this case. 
An inspection of the proof of Theorem 5.1 shows that the assertion of this result holds for any $\alpha<\gamma_{*}$. Therefore we have

Corollary 5.2. Let the assumptions of Theorem 3.1 be satisfied, and suppose $\int_{0}^{\infty} e^{\alpha_{0} t} k(t) d t<\infty$, for some $\alpha_{0}>0$.

Then the optimal decay rate $\gamma_{*}>0$ for the operator families $S(t), A^{1 / 2} R(t)$, $\dot{S}(t) A^{-1 / 2}$ and $\ddot{S}(t) A^{-1}$ is given by $\gamma_{*}=\min \left\{\alpha_{1}, \alpha_{2}\right\}$, where $\alpha_{1}$ and $\alpha_{2}$ are defined in (5.2) and (5.3).

6. A semilinear problem. In this section we briefly consider the semilinearly perturbed problem

$$
\begin{aligned}
\ddot{u}(t)+A u(t)-(k * A u)(t) & =F(u(t), \dot{u}(t)), \quad t>0, \\
u(0)=u_{0}, \quad \partial_{t} u(0) & =u_{1} .
\end{aligned}
$$

Here $X, A, k$ are as before, and $F: D\left(A^{1 / 2}\right) \times X \rightarrow X$ is Lipschitz with sufficently small Lipschitz constant in a neighborhood of 0 , and $F(0)=0$. Thus the principal linearization of (6.1) is the linear problem (1.1).

The basic idea to solve (6.1) is of course to use the contraction mapping principle. By the stability properties of the linearized problem, this yields immediately global solutions which tend to 0 in energy norm as $t \rightarrow \infty$, provided the initial values are small in this norm. For this, one only has to know the following result which is valid in general Banach spaces.

Lemma 6.1. Let $X, Y$ be Banach spaces, and let $\{U(t)\}_{t \geq 0} \subset \mathcal{B}(X, Y)$ be a strongly continuous operator family which is such that $U(\cdot)$ as well as $U^{*}(\cdot)$ are strongly integrable. Then the convolution operator $T$ defined by

$$
(T f)(t):=\int_{0}^{t} U(t-s) f(s) d s, \quad t \geq 0,
$$

is well-defined and bounded from $L_{p}\left(\mathbb{R}_{+} ; X\right)$ into $L_{p}\left(\mathbb{R}_{+} ; Y\right)$, for each $p \in[1, \infty]$. $T$ is also bounded from $C_{0}\left(\mathbb{R}_{+} ; X\right)$ into $C_{0}\left(\mathbb{R}_{+} ; Y\right)$, provided $U(t) \rightarrow 0$ strongly as $t \rightarrow \infty$.

The proof of this result follows by standard arguments as in [8, p. 257, 273]. The last assertion is a consequence of the fact that $f \in C_{0}\left(\mathbb{R}_{+} ; X\right)$ can be uniformly approximated by simple functions with compact support.

Let the assumptions of Theorem 3.1 be satisfied. Then the operator families $A^{1 / 2} R(t)$ and $S(t)$ are strongly continuous, strongly integrable and converge to 0 strongly as $t \rightarrow \infty$. We rewrite problem (6.1) as

(6.2) $u(t)=u_{*}(t)+\int_{0}^{t} R(t-s) F(u(s), \dot{u}(s)) d s, u_{*}(t)=S(t) u_{0}+R(t) u_{1}, t \geq 0$. 
As the basic space for the fixed point argument, we choose $Z=C_{0}^{1}\left(\mathbb{R}_{+} ; X\right) \cap$ $C_{0}\left(\mathbb{R}_{+} ; D\left(A^{1 / 2}\right)\right)$, equipped with the norm

$$
\|u\|_{Z}=\sup _{t \geq 0}|u(t)|_{E}, \quad|u(t)|_{E}:=\left|A^{1 / 2} u(t)\right|_{X}+|\dot{u}(t)|_{X},
$$

and we let $Y:=C_{0}\left(\mathbb{R}_{+} ; X\right)$ with norm $\|f\|_{Y}:=\sup _{t \geq 0}|f(t)|$. If $u_{0} \in D\left(A^{1 / 2}\right)$ and $u_{1} \in X$ we have $u_{*} \in Z$. Next suppose that $F: E \rightarrow X$ is Lipschitz on each ball $\bar{B}_{r}(0,0) \subset E:=D\left(A^{1 / 2}\right) \times X, r \leq R$, say with constant $L(r)$. Define $\bar{F}$ by means of

$$
\bar{F}(u)(t):=F(u(t), \dot{u}(t)), \quad t \geq 0, u \in Z .
$$

Then $\bar{F}: Z \rightarrow Y$ is Lipschitz on each ball $B_{r}(0) \subset Z$ as well, with the same constant $L(r)$. Now fix a ball $\mathbb{B}:=\bar{B}_{\rho}\left(u_{*}\right) \subset Z$, with $\rho \leq r$, and define the map $T: \mathbb{B} \rightarrow Z$ by means of the right hand side of (6.2), i.e.

$$
(T u)(t):=u_{*}(t)+\int_{0}^{t} R(t-s) \bar{F}(u)(s) d s, \quad t \geq 0, u \in \mathbb{B} .
$$

Since $R(t)$ is selfadjoint in $X$, by Theorem 3.1 and Lemma 6.1, $T$ is well-defined, and there is a constant $C>0$ such that

$$
\|R * f\|_{Z} \leq C\|f\|_{Y}, \quad \text { for each } f \in Z .
$$

Therefore we obtain

$$
\|T u-T v\|_{Z} \leq C L(r)\|u-v\|_{Z}, \quad \text { for all } u, v \in B_{r}(0),
$$

as well as

$$
\left\|T u-u_{*}\right\|_{Z} \leq C L(r)\|u\|_{Z} \leq C L(r)\left(\rho+\left\|u_{*}\right\|_{Z}\right), \quad u \in \mathbb{B} .
$$

Therefore $T$ is strict contraction provided say $C L(r) \leq 1 / 2$, and it maps $\mathbb{B}$ into $\mathbb{B}$, provided in addition $\rho:=\left\|u_{*}\right\|_{Z} \leq r / 2$, i.e. if $\left|A^{1 / 2} u_{0}\right|$ and $\left|u_{1}\right|$ are small enough. Thus the contraction principle applies and we have a unique global solution of (6.2) which is continuous and converges to 0 as $t \rightarrow \infty$ in energy norm, provided $L(r)$ and $\left|A^{1 / 2} u_{0}\right|$ and $\left|u_{1}\right|$ are small enough. This shows that the trivial solution of the semilinear problem (6.1) is asymptotically stable in energy norm.

To obtain decay rates, suppose that the assumptions of Theorem 5.1 are valid, and let $u$ be a solution of (6.2). Then setting $\phi(t)=|u(t)|_{E}$ we obtain from the integral equation (6.2) the estimate

$$
\phi(t) \leq M e^{-\alpha t} \phi(0)+M L(r) \int_{0}^{t} e^{-\alpha(t-s)} \phi(s) d s,
$$

hence

$$
\phi(t) \leq M e^{-(\alpha-M L(r)) t} \phi(0), \quad t \geq 0,
$$


provided $\alpha>M L(r)$, i.e. provided $L(r)$ is sufficiently small. Similarly, if the assumptions of Theorem 4.1 hold, then setting $\kappa(t)=(1+t)^{-m}$ we obtain from $(6.2)$

$$
\phi(t) \leq M \kappa(t) \phi(0)+M L(r) \int_{0}^{t} \kappa(t-s) \phi(s) d s, \quad t \geq 0 .
$$

Let the resolvent kernel $\rho(t)$ be defined by

$$
\rho(t)=\kappa(t)+M L(r)(\kappa * \rho)(t) \quad t \geq 0,
$$

by the Neumann series we have $0 \leq \rho \in L_{1}\left(\mathbb{R}^{+}\right)$provided $M L(r) \widehat{\kappa}(0)<1$, which means $M L(r)<m-1$. Convolving (6.3) with $M L \rho$ and adding the result to (6.3) we obtain

$$
\phi(t) \leq M \rho(t) \phi(0), \quad t \geq 0 .
$$

Since $\kappa(t)$ is positive and decreasing,

$$
(\kappa * \kappa)(t) \leq 2 \widehat{\kappa}(0) \kappa(t / 2) \leq \frac{2^{m+1}}{m-1} \kappa(t),
$$

by induction and the Neumann series for $\rho$ we obtain

$$
\rho(t) \leq \kappa(t) \sum_{j=0}^{\infty}\left[\frac{M L 2^{m+1}}{m-1}\right]^{j}=\kappa(t)\left[1-\frac{M L 2^{m+1}}{m-1}\right]^{-1},
$$

provided $m \geq 2$ and $L(r)$ is sufficiently small. This way we obtain a decay like $t^{-m}$ of the solution in energy norm.

Summarizing we have

Theorem 6.2. Let the assumptions of Theorem 3.1 hold, let $E$ be the energy space $E=D\left(A^{1 / 2}\right) \times X$, and assume that $F: E \rightarrow X$ is Lipschitz with constant $L(r)$ on the ball $B_{r}(0) \subset E, F(0)=0$ and suppose $L(r) \rightarrow 0$ as $r \rightarrow 0$.

Then there is a constant $r_{0}>0$ such that (6.1) has a unique solution

$$
u \in C_{0}\left(\mathbb{R}_{+} ; D\left(A^{1 / 2}\right)\right) \cap C_{0}^{1}\left(\mathbb{R}_{+} ; X\right),
$$

provided $\left|A^{1 / 2} u_{0}\right|_{X}+\left|u_{1}\right|_{X} \leq r_{0}$. Moreover,

(i) if $\int_{0}^{\infty} t^{m} k(t) d t<\infty$ for some $m \geq 2$, then

$$
\left|A^{1 / 2} u(t)\right|_{X}+|\dot{u}(t)|_{X} \leq C(1+t)^{-m} \quad t \geq 0 ;
$$

(ii) if $\int_{0}^{\infty} e^{\alpha_{0} t} k(t) d t<\infty$ for some $\alpha_{0}>0$ then

$$
\left|A^{1 / 2} u(t)\right|_{X}+|\dot{u}(t)|_{X} \leq C e^{-\alpha t} \quad t \geq 0,
$$

for some $\alpha>0$. Here $C>0$ is a constant which is independent of the solution $u(t)$.

Obvious modifications of the arguments given above show that the nonlinear function $F$ can be replaced by a memory dependent but causal functional. We leave this and other possible extensions to the interested reader. 
Acknowledgement. I would like to thank the anonymous referee for his careful reading and for his useful comments which lead to some improvements in the paper.

\section{References}

[1] F. Alabau-Boussouira, P. Cannarsa, and D. Sforza, Decay estimates for second order evolution equations with memory, J. Funct. Anal. 254, 1342-1372 (2008).

[2] J. A. D. Appleby et AL., On exponential asymptotic stability in linear viscoelasticity, Math. Models Methods Appl. Sci 16, 1677-1694 (2006).

[3] S. Berrini and S. A. Messaoudi, Existence and decay of solutions of a viscoelastic equation with a nonlinear source, Nonlin. Anal. 64, 2314-2331 (2006).

[4] E. FAsÁngova AND J. PrÜss, Evolution equations with dissipation of memory type. Topics in Nonlinear Analysis, Birkhäuser Verlag, Basel 1998

[5] E. FAsángova And J. Prüss, Asymptotic behaviour of a semilinear viscoelastic beam model, Arch. Math. 77, 488-497 (2001).

[6] J. E. Munoz Rivera And E. C. LAPA, Decay rates of solutions of an anisotropic inhomogeneous $n$-dimensional viscoelastic equation with polynomially decaying kernels, Comm. Math. Physics 177, 583-602 (1996).

[7] J. E. Munoz Rivera and E. C. LAPA, Decay rates for viscoelastic plates with memory, J. Elasticity 44, 61-87 (1996).

[8] J. PRÜss, Evolutionary Integral Equations and Applications, Monographs in Mathematics 87, Birkhäuser, Basel, 1993.

JAN PRÜss, Institut für Mathematik, Fakultät NW III, Martin-Luther-Universität HalleWittenberg, Theodor-Lieser-Str. 5, D-06120 Halle, Germany

e-mail: jan.pruess@mathematik.uni-halle.de

Received: 9 July 2008

Revised: 16 September 2008 